\title{
EDITORIAL
}

\section{LA INVESTIGACIÓN SOBRE TABAQUISMO EN ESPAÑA}

\author{
Ana Álvarez Requejo \\ Consejería de Sanidad y Bienestar Social. Junta de Castilla y León. Valladolid
}

La situación de la investigación publicada sobre los diferentes aspectos del tabaquismo en España reflcja, en bucna medida, cl cstado en que se encuentra cada uno de estos dos temas: la investigación en ciencias de la salud, por un lado, y los conocimientos sobre las características y el alcance del consumo de tabaco, así como las medidas de prevención y control del tabaquismo, por otro.

Se ha definido al tabaquismo como la gran epidemia silenciosa del siglo XX y, sin duda, supone actualmente uno de los mayores problemas de salud pública a escala mundial. Un problema que surgió claramente asociado al desarrollo, la industrialización y la influencia de los valores materiales predominantes en la sociedad de consumo, que afecta actualmente a una porción enorme de la población y que ocasiona tres millones de muertes al año, con una tendencia creciente, de manera que se estima que en el año 2020 la mortalidad atribuible al tabaco superará los de 8 millones anuales de muertes '.

Al mismo tiempo, este siglo xx que está a punto de concluir ha producido los mayores avances en el terreno de la investigación y el desarrollo científico y tecnológico, entre ellos en investigación biomédica, incluida la de salud pública. La epidemiología, materia básica del conocimiento científico de la salud pública, ha experimentado un desarrollo y consolidación notables en la segunda mitad del siglo. Han pasado casi 50 años desde que Doll y Hill ${ }^{2}$ mostraron por primera vez, mediante un estudio de casos y controles, la cvidencia de esa relación. Multitud de estudios posteriores han ratificado este hecho y han puesto de manifiesto otros múltiples riesgos que entraña el tabaco, hasta el extremo de que pocos factores habrán sido a lo largo de estos años más desmenuzados, examinados, analizados y encontrados implicados en relación con un amplio espectro de enfermedades, principalmente cánceres, patologías respiratorias y cardiovasculares.

Resulta paradójico que uno de los hallazgos más precoces, consistentes e irrefutables que ha proporcionado la epidemiología moderna, la evidencia de la asociación causal existente entre el hábito de fumar tabaco y el cáncer de pulmón, no haya conseguido modificar de forma más sustancial el consumo de tabaco. Para mejorar la salud, es preciso que los avances de los conocimientos científicos se traduzcan el acciones positivas. En este punto es donde la epidemiología moderna ha sido criticada de preocuparse más de los procedimientos metodológicos y «tecnicismos» que de los problemas que analiza y también se ha puesto de relieve la necesidad de que los epidemiólogos se impliquen más estrechamente en la aplicación práctica de sus descubrimientos ${ }^{3}$.

La corriente cada vez más potente y extendida de la medicina basada en la evidencia ${ }^{4}$ ha contribuido al rápido desarrollo y afianzamiento de las medidas necesarias para 
la aproximación entre la ciencia y la práctica clínica. Sin embargo, en el terreno de la salud pública el recorrido para avanzar en esta aproximación se está mostrando más largo y tortuoso que en el clínico, posiblemente debido a la naturaleza del campo de actuación ${ }^{5}$, en el que influyen otras disciplinas además de la medicina, como la economía, sociología, demografía o pedagogía, además de poderosos intereses políticos y económicos. Es patente que hasta la fecha los aspectos médicos y de salud pública no parecen ser los que más peso han tenido en relación con el tabaquismo, sino que están influyendo de forma más importante razones económicas, sociales y políticas. Ante otros factores de riesgo con los mismos o menos «tantos en su contra» como el colesterol, la hipertensión o incluso el sedentario se han conseguido mejores resultados, en cambio, con el tabaco la progresión está resultando más lenta y llena de altibajos en su trayectoria.

También tiene plena vigencia en relación con el tabaquismo el debate sobre las ventajas e inconvenientes entre métodos de investigación cuantitativos y cualitativos en salud pública ${ }^{6}$. En el consumo de tabaco y sus consecuencias, los factores psicológicos, sociales, políticos, económicos y ambientales son al menos tan importantes como los genéticos, biológicos y médicos. Por ello parece incuestionable que para avanzar en la reducción del tabaquismo los métodos de estudio, los factores que se consideren, y las intervenciones que se apliquen, habrán de ser creativos y considerar una variedad de alternativas complementarias a las que se han mostrado eficaces hasta el momento, además de tener en cuenta los factores culturales, ambientales y peculiaridades propias del país al que se dirija ${ }^{7}$.

En los últimos 15-20 años, la investigación científica en general y la biomédica en particular han experimentado un notable desarrollo en España,si bien no ha sido suficiente para remontar el atraso de muchas décadas que manteníamos respecto a otros países europeos próximos. El letargo que impuso a la actividad científica y cultural la Guerra Civil y la depresión económica consecuente, así como el aislamiento externo posterior, influyó en este atraso y también en que la epidemia de tabaquismo llegase a España con varias décadas de retraso respecto a países como Estados Unidos, el Reino Unido o los del centro y norte de Europa. También hemos tardado en reaccionar $y$, aunque la situación parece encontrarse en plena transición, todavía hoy en día el hábito de fumar es socialmente bien aceptado es España

Este número de la Revista de Salud Pública, que coincide con la celebración el 31 de mayo del Día Mundial sin Tabaco, dedica una buena parte de su contenido al tabaquismo en España. El trabajo de García López ${ }^{8}$ muestra una producción científica sobre tabaquismo publicada desde 1985 a 1996 en cuatro revistas españolas de magnitud considerable (el 4\% de los artículos originales se referían a tabaquismo), pero de contenido bastante elemental. La mayoría eran estudios descriptivos relativos a prevalencias de uso en diversas áreas geográficas, así como hábitos de uso, actitudes, conocimientos y conductas frente al tabaquismo. Los estudios descriptivos son el primer paso en la secuencia de aproximación a un tema ${ }^{9}$, para avanzar en el conocimiento de sus causas son necesarios estudios observacionales analíticos y para valorar la eficacia de las intervenciones de control, estudios experimentales. Es posible que si en el trabajo se hubiesen incluido también los artículos publicados en alguna otra revista española con una orientación de salud pública $\mathrm{y}$, sobre todo, los publicados en revistas extranjeras (presumiblemente los trabajos con diseños más sofisticados y de mayor calidad se habrán dirigido a revistas con mayor impacto, en su mayoría en lengua inglesa) el panorama fuese un poco diferente, pero no muy distinto del que presenta García López. De hecho, sus resultados son coincidentes con los de otros trabajos que han analizado mediante técnicas bibliométricas la investigación publicada en España sobre diversas 
especialidades médicas y de salud públi$\mathrm{ca}^{10}$.

Otros dos artículos de este número tratan el tabaquismos en dos áreas especialemente relevantes: las mujeres profesionales de la salud " ${ }^{11}$ los programas de prevención extraescolares dirigidos a adolescentes ${ }^{12}$. La prevalencia de consumo de tabaco en médicas y enfermeras de la Comunidad de Madrid en 1998 que encuentran Fernández Ruiz y cols. ${ }^{11}$ es del $43 \%$, ligeramente más alta que el 39\% referido para el conjunto de profesionales correspondientes al territorio Insalud en el estudio elaborado por el Ministerio de Sanidad y Consumo ${ }^{13}$. Cuando se desglosan los resultados en función de género y profesión, ambos estudios son coincidentes y muestran una mayor prevalencia de fumadores entre las mujeres, mucho más alta en el personal de enfermería y especialmente en el grupo de edad más joven. Estos resultados parecen reflejar un una creciente sensibilización del personal médico, al que inicialmente se dirigieron las intervenciones, y la necesidad de realizar actividades desensibilización y programas de ayuda para dejar de fumar dirigidos al personal de enfermería, colectivo compuesto mayoritariamente por mujeres jóvenes, que actualmente son el grupo de población con la prevalencia más elevada de fumadores en España.

La perspectiva actual del tabaquismo en España desgraciadamente no es halagüeña, pero tampoco desesperada. Se han producido notables avances y se perciben con claridad aspectos positivos, como son la disminución progresiva del número de fumadores; la ampliación de las restricciones a la venta, consumo y promoción del tabaco mediante medidas legislativas tanto en las Comunidades Autónomas como de ámbito estatal; la extensión de los programas y actividades estructuradas de consejo y ayuda para dejar fumar en la atención primaria y especializada o la aplicación cada vez más frecuente de programas preventivos en la escuela. Al lado de estos cambios positivos, son tangibles importantes deficiencias, fun- damentalmente en cuanto se refiere a la prohibición de la publicidad directa e indirecta de productos de tabaco, incluyendo la promoción y patrocinio de actividades deportivas, recreativas y culturales; la restricción del acceso de los menores al de tabaco, especialmente a través de las máquinas expendedoras automáticas y la venta de cigarrillos sueltos; la falta de garantía del derecho a respirar un aire libre de tabaco en los lug ares de uso público, junto con una gran laxitud en el cumplimiento de la legislación respecto a las limitaciones a la venta y consumo de tabaco.

Esta situación muestra la necesidad de potenciar la investigación sobre tabaquismo, fundamentalmente la de tipo analítico y experimental, con métodos innovadores y en particular en áreas menos exploradas hasta el momento, como pueden ser nuevas opciones para comprender por qué los adolescentes y jóvenes empiezan a fumar, así como estrategias eficaces para su prevención y métodos apropiados para ayudar a dejar de fumar a los jóvenes; percepción de riesgos y su influencia en los hábitos de vida, intervenciones para modificar dichas percepciones; la posible influencia de los medios de comunicación; el empleo de medidas de movilización social o las intervenciones comunitarias en el control del tabaquismo. Asimismo, se percibe claramente que es necesaria una actuación pública mucho más decidida en la limitación de la exposición que reciben los menores al influjo del tabaco, principalmente a través de la publicidad y del fácil acceso al tabaco, y en garantizar el derecho de todos a respirar aire sin humo en los espacios de uso público.

\section{BIBLIOGRAFÍA}

1. Murray CJ; López AD. Alternative projections of mortality and disability by cause 1990-2020: global burden of disease study. Lancet 1997; 349: 1498-1504.

2. Doll R, Hill AB. Smoking and carcinoma of the lung: preliminary report.BMJ 1952; 2: 739-748. 
3. Colditz GA. Epidemiology: future directions. Int J Epidemiol. 1997; 26:693-697.

4. Sackett DL, Rosnberg WMC, Gary JAM, Haynes RB, Richardson WS. Evidence based medicine: what is it and what it isn't. BMJ 1996; 312: 71-72.

5. Rodríguez Artalejo F. La salud pública basada en la evidencia. Gac Sanit 1997; 11: 201-203.

6. Baum F. Investigación en salud pública: el debate sobre las metodologías cuantitativas y cualitativas. Revisiones en Salud Pública 1997; 5: 175-193.

7. Samet JM, Taylor CE, Becker KM, Yach D. Research in support of tobacco control. BMJ 1988; 316: 321.

8. Garcia López JA. Metodología empleada en los artículos originales publicados sobre tabaquismo en cuatro revistas médicas españolas (1985-1996). Rev Esp Salud Pública 1999; 73:333-341.

9. Hulley SB, Newman TB, Cummings SR. Getting started: the anatomy and physiology of research.
En: Hulley SB, Cummings SR. Designing clinical research: an epidemiological approach. Baltimore: Williams \& Wilkins, 1988 p: 1-11.

10. Álvarez Solar M, López González ML, Cueto Espinar A. Indicadores bibliométricos, anaálisis temático y metodológico de la investigación publicada cn España sobre epidemiología y salud pública (1988-1992). Med Clin (Barc) 1998; 111 : 529-535.

11. Fernández Ruiz ML, Sánchez Bayle M. Prevalencia de consumo de tabaco entre las médicas y enfermeras de la Comunidad de Madrid. Rev Esp Salud Pública 1999; 73:355-364.

12. López González ML, López T, Comas Fuentes A, Herrero Puente P, González Blázquez J, Cueto Espinar A et al., por el Equipo OCTOPUS. Actividades extraescolares de los adolescentes útiles para programas de prevención del tabaquismo. Rev Esp Salud Pública 1999; 73:343-353.

13. Ministerio de Sanidad y Consumo. Prevalencia de consumo de tabaco en los profesionales sanitarios del Insalud 1998, España [informe preliminar]. Madrid: Ministerio de Sanidad y Consumo; 1999. 